\title{
World-Volume Effective Theory for Higher-Dimensional Black Holes
}

\author{
Roberto Emparan, ${ }^{1,2, *}$ Troels Harmark, ${ }^{3, \dagger}$ Vasilis Niarchos, ${ }^{4,+}$ and Niels A. Obers ${ }^{3, \S}$ \\ ${ }^{1}$ Institució Catalana de Recerca i Estudis Avançats (ICREA), Passeig Lluis Companys 23, 08010 Barcelona, Spain \\ ${ }^{2}$ Departament de Física Fonamental, Universitat de Barcelona, Marti i Franquès 1 E-08028 Barcelona, Spain \\ ${ }^{3}$ The Niels Bohr Institute, Blegdamsvej 17, 2100 Copenhagen $\emptyset$, Denmark \\ ${ }^{4}$ Centre de Physique Théorique-École Polytechnique, Unité mixte de Recherche 7644, CNRS, 91128 Palaiseau, France
}

(Received 9 February 2009; published 11 May 2009)

\begin{abstract}
We argue that the main feature behind novel properties of higher-dimensional black holes, compared to four-dimensional ones, is that their horizons can have two characteristic lengths of very different size. We develop a long-distance world-volume effective theory that captures the black hole dynamics at scales much larger than the short scale. In this limit the black hole is regarded as a blackfold: a black brane (possibly boosted locally) whose world volume spans a curved submanifold of the spacetime. This approach reveals black objects with novel horizon geometries and topologies more complex than the black ring, but more generally it provides a new organizing framework for the dynamics of higher-dimensional black holes.
\end{abstract}

DOI: 10.1103/PhysRevLett.102.191301

PACS numbers: 04.50.Gh, 11.25.Wx, 11.27.+d

It has been realized in recent years that the dynamics of black holes in spacetimes of dimension $D \geq 5$ is much richer than in four dimensions. While the techniques developed to construct and characterize four-dimensional black holes have been quite successful in five dimensions, the dynamics in $D \geq 6$ seems to be too complex to be captured by conventional approaches.

The main novel feature of higher-dimensional neutral black holes is that in some regimes their horizons are characterized by at least two separate scales, $r_{0} \ll R$. This does not occur in four dimensions, where the shape of a Kerr black hole is always approximately round with radius $r_{0} \sim G M$. In particular the angular momentum bound $J \leq G M^{2}$ implies that rotation cannot produce large distortions on the horizon. However, in $D \geq 5$ such bounds do not generally hold and the two classical length scales $J / M$ and $(G M)^{1 /(D-3)}$ can be widely separated, as we know from explicit solutions. Five-dimensional black rings can have arbitrarily large angular momentum for a given mass, and at large $J$ the ring's radius $R$ is much bigger than its thickness $r_{0}$ [1]. Such black rings are also well approximated by (boosted) black strings. Likewise, in $D \geq 6$ Myers-Perry black holes have ultraspinning regimes with pancaked horizons approaching black membranes of small thickness $r_{0}$ and large extent $R$ along the plane of rotation $[2,3]$. There are other phenomena peculiar to higherdimensional horizons that depend on the ability to separate two length scales along the horizon: the GregoryLaflamme instability and its associated inhomogeneous black branes [4] arise when the two scales characterizing the thickness and the length of a black brane begin to differ. In hindsight, it is surprising that four-dimensional black hole horizons only possess short-scale $\left(\sim r_{0}\right)$ dynamics. Thus it is clear that new tools are needed in order to capture the long-distance $\left(\sim R \gg r_{0}\right)$ dynamics of higherdimensional horizons.
The natural approach when faced with a problem with two widely separate length scales is to integrate out the short-distance physics to obtain a long-distance effective theory. In general relativity there are two different (but essentially equivalent) techniques to do this: matched asymptotic expansions [5] and the classical effective field theory of [6]. To the order that we work in this Letter there is no difference between them. We shall refer to this leading-order theory for the long-distance dynamics of higher-dimensional black holes as the blackfold approach. This program was initiated in [7] with the construction of thin black rings in $D \geq 5$. Here we present a general framework.

For spacetime dimension $D$ and spatial dimension $p$ of the blackfold world volume, we denote $n=D-p-3$. Choosing units where $16 \pi G=\Omega_{n+1}$ simplifies some equations.

A blackfold is a black p-brane whose world volume extends along a curved submanifold of the embedding spacetime. Beginning from a flat black $p$-brane with horizon $\mathbb{R}^{p} \times s^{n+1}$ (we denote the sphere of short-size $r_{0}$ with lowercase $s$ ), we imagine bending its world volume $\mathbb{R}^{p, 1}$ into some submanifold $\mathcal{W}_{p+1}$, with spatial section $\mathcal{B}_{p}$ compact or not, characterized by a length scale (e.g., intrinsic curvature radius) $R \gg r_{0}$. Describing the longdistance dynamics of the blackfold amounts to finding the spacetime embedding $X^{\mu}\left(\sigma^{\alpha}\right)$ of its world volume. This embedding determines the induced (pulled-back) metric, $\gamma_{\alpha \beta}$, and the first fundamental tensor $h_{\mu \nu}$, which acts as a projector onto the world volume,

$$
\gamma_{\alpha \beta}=g_{\mu \nu} \partial_{\alpha} X^{\mu} \partial_{\beta} X^{\nu}, \quad h^{\mu \nu}=\gamma^{\alpha \beta} \partial_{\alpha} X^{\mu} \partial_{\beta} X^{\nu} .
$$

A general theory of the classical dynamics of a branelike object, regarded as a source of energy-momentum $T_{\mu \nu}$ in the probe approximation, was developed in [8]. The consistent coupling of the source to gravity requires 
$\nabla_{\mu} T^{\mu \nu}=0$. This implies that

$$
T^{\mu \nu} K_{\mu \nu}^{\rho}=0,
$$

where $K_{\mu \nu}^{\rho}$ is the extrinsic curvature tensor of the blackfold embedding,

$$
K_{\mu \nu}^{\rho}=h_{\mu}^{\lambda} h_{\nu}^{\sigma} \nabla_{\lambda} h_{\sigma}^{\rho},
$$

with indices $\mu, \nu$ along directions tangent to the brane world volume and $\rho$ orthogonal to it.

The effective stress-energy tensor of a blackfold is determined by matching to short-distance physics. We demand that locally, i.e., on scales $r \ll R$, the blackfold be equivalent to a black $p$-brane up to a position-dependent Lorentz transformation. The gravitational field of such a black $p$-brane is known, and at distances much larger than its thickness, $r \gg r_{0}$, the field is weak. Thus, we can determine an equivalent distributional stress tensor

$$
T_{\mu \nu}\left(\sigma^{\alpha}\right)=\tau_{\mu \nu}\left(\sigma^{\alpha}\right) \delta^{(D-p-1)}\left(x-X\left(\sigma^{\alpha}\right)\right)
$$

that sources the same linearized field in the matching range $r_{0} \ll r \ll R$.

In [9] we show that the blackfold Eqs. (2) are equivalent to a generalized geodesic equation

$$
\tau^{\alpha \beta}\left(\nabla_{\alpha}^{(\gamma)} \partial_{\beta} X^{\rho}+\Gamma_{\mu \nu}^{\rho} \partial_{\alpha} X^{\mu} \partial_{\beta} X^{\nu}\right)=0
$$

and also that they can be derived by coupling the worldvolume action

$$
I\left[X^{\mu}\left(\sigma^{\alpha}\right)\right]=\int_{\mathcal{W}_{p+1}} d V_{p+1} \tau^{\alpha \beta} \gamma_{\alpha \beta}
$$

to bulk gravity.

The effective stress tensor $\tau_{a b}$ of a static flat black $p$-brane with orthonormal world-volume coordinates $z^{a}=$ $\left(z^{0}, z^{i}\right)$ is

$$
\tau_{00}=r_{0}^{n}(n+1), \quad \tau_{i i}=-r_{0}^{n},
$$

where $r_{0}$ is the horizon radius in directions transverse to the world volume, i.e., the thickness of the black $p$-brane.

Consider now a Lorentz transformation $\Lambda \in$ $S O(1, m) \subset S O(1, p)$ of the $p$-brane, where $m \leq p$ is the number of directions along which the blackfold is boosted, and which cannot be larger than the number of independent rotation planes of the spacetime. We parametrize the $m$ boosts as

$$
\Lambda_{0}^{0}=\cosh \alpha, \quad \Lambda_{i}^{0}=\nu_{i} \sinh \alpha, \quad \sum_{i=1}^{m} \nu_{i}^{2}=1,
$$

where $\nu_{i}$ are the director cosines of a $S^{m-1}$ in parameter space. The remaining components of $\Lambda$ are constrained by $\left(\Lambda \eta \Lambda^{T}\right)_{a b}=\eta_{a b}$, but are otherwise irrelevant.

We parametrize the background spacetime using coordinates $\left(t, r_{1}, \phi_{1}, \ldots, r_{m}, \phi_{m}, x_{1}, \ldots, x_{D-1-2 m}\right)$, where $\left(r_{l}\right.$, $\phi_{l}$ ) are polar coordinates for the $l$ th rotation plane, with $r_{l}$ measuring proper distance along the orbits of $\partial_{\phi_{l}}$. The embedding is specified by

$$
X^{\mu}\left(\sigma^{\alpha}\right)=\left(t\left(\sigma^{\alpha}\right), r_{l}\left(\sigma^{\alpha}\right), \phi_{l}\left(\sigma^{\alpha}\right), x_{k}\left(\sigma^{\alpha}\right)\right) .
$$

We specialize from now on to static backgrounds and stationary blackfolds, i.e., we align $t \propto z^{0}=\sigma^{0}$ and take $X^{i}$ independent of $\sigma^{0}$. We assume that $\partial_{\phi_{l}}$ generate isometries of the background and align the $m$ boosted spatial coordinates on the $p$-brane with the angular directions $\phi_{l}$. The $m$ boosted spatial coordinates are thus identified periodically as $z^{l} \sim z^{l}+2 \pi r_{l}\left(\sigma^{\alpha}\right)$.

In a blackfold the thickness and boost parameters in general depend on the position, $r_{0}\left(\sigma^{\alpha}\right), \alpha\left(\sigma^{\alpha}\right), \nu_{i}\left(\sigma^{\alpha}\right)$. However, to ensure regularity of the horizon we impose a blackness condition: the surface gravity $\kappa$ and the angular velocities $\Omega_{\mathrm{H} i}$ must be uniform over $\mathcal{B}_{p}$. Locally, these are determined by the horizon properties of a boosted black p-brane,

$$
\kappa=\frac{n}{2 r_{0}\left(\sigma^{\alpha}\right) \cosh \alpha\left(\sigma^{\alpha}\right)}, \quad \Omega_{\mathrm{H} i}=\frac{\nu_{i}\left(\sigma^{\alpha}\right)}{r_{i}\left(\sigma^{\alpha}\right)} \tanh \alpha\left(\sigma^{\alpha}\right) .
$$

Requiring blackness determines the thickness and boosts in terms of the local velocity components $r_{i}\left(\sigma^{\alpha}\right) \Omega_{\mathrm{H} i}$,

$$
\begin{gathered}
\tanh \alpha\left(\sigma^{\alpha}\right)=\Xi\left(\sigma^{\alpha}\right), \quad r_{0}\left(\sigma^{\alpha}\right)=\frac{n}{2 \kappa} \sqrt{1-\Xi\left(\sigma^{\alpha}\right)^{2}}, \\
\nu_{i}\left(\sigma^{\alpha}\right)=\frac{r_{i}\left(\sigma^{\alpha}\right) \Omega_{\mathrm{H} i}}{\Xi\left(\sigma^{\alpha}\right)},
\end{gathered}
$$

where the local velocity field is

$$
\Xi\left(\sigma^{\alpha}\right)=\left[\sum_{i=1}^{m}\left(r_{i}\left(\sigma^{\alpha}\right) \Omega_{\mathrm{H} i}\right)^{2}\right]^{1 / 2} .
$$

Boosting $z^{a} \rightarrow(\Lambda z)^{a}, \tau_{a b} \rightarrow\left(\Lambda \tau \Lambda^{T}\right)_{a b}$ we obtain

$$
\begin{gathered}
\tau_{00}=\left(\frac{n}{2 \kappa}\right)^{n}\left(1-\Xi^{2}\right)^{(n-2) / 2}\left(n+1-\Xi^{2}\right), \\
\tau_{0 i}=\left(\frac{n}{2 \kappa}\right)^{n}\left(1-\Xi^{2}\right)^{(n-2) / 2} n r_{i} \Omega_{\mathrm{H} i}, \quad i=1, \ldots, m, \\
\tau_{i i}=\left(\frac{n}{2 \kappa}\right)^{n}\left(1-\Xi^{2}\right)^{n / 2}\left(\frac{n\left(r_{i} \Omega_{\mathrm{H} i}\right)^{2}}{1-\Xi^{2}}-1\right), \quad i=1, \ldots, m, \\
\tau_{i i}=-\left(\frac{n}{2 \kappa}\right)^{n}\left(1-\Xi^{2}\right)^{n / 2}, \quad i=m+1, \ldots, p, \quad(16) \\
\tau_{i \neq j}=\left(\frac{n}{2 \kappa}\right)^{n}\left(1-\Xi^{2}\right)^{(n-2) / 2} r_{i} r_{j} \Omega_{\mathrm{H} i} \Omega_{\mathrm{H} j}, \quad i, j=1, \ldots, m .
\end{gathered}
$$

As a consequence of the blackness condition, once the $\Omega_{\mathrm{H} i}$ are given the blackfold Eq. (2) becomes a purely geometric one involving only $X^{\mu}\left(\sigma^{\alpha}\right)$ and its first and second derivatives. $\kappa$ factorizes out of the equations and enters only to fix the horizon thickness $r_{0}\left(\sigma^{\alpha}\right)$. 
The mass and angular momenta of the blackfold are obtained by integrating the energy and momentum densities over $\mathcal{B}_{p}$, on which $\partial_{t}=N \partial_{z^{0}}$ ( $N$ accounts for a possible redshift between the blackfold and infinity) and $\partial_{\phi_{i}}=r_{i} \partial_{z^{i}}$. Then

$$
M=\int_{\mathcal{B}_{p}} d V_{p} N \tau_{00}, \quad J_{i}=\int_{\mathcal{B}_{p}} d V_{p} r_{i} \tau_{0 i}
$$

At each point on $\mathcal{B}_{p}$ we assume the presence of a small sphere $s^{n+1}(n \geq 1)$ locally equal to that in a boosted black $p$-brane and so with area

$$
\begin{aligned}
a_{\mathrm{H}}\left(\sigma^{\alpha}\right) & =\Omega_{n+1} r_{0}^{n+1}\left(\sigma^{\alpha}\right) \cosh \alpha\left(\sigma^{\alpha}\right) \\
& =\Omega_{n+1}\left(\frac{n}{2 \kappa}\right)^{n+1}\left(1-\Xi^{2}\right)^{n / 2} .
\end{aligned}
$$

The horizon of the blackfold is therefore a fibration of $s^{n+1}$ over $\mathcal{B}_{p}$. If the fiber is regular everywhere then the topology of the horizon is (topology of $\mathcal{B}_{p}$ ) $\times S^{n+1}$. However, the size $r_{0}\left(\sigma^{\alpha}\right)$ will decrease to zero at "walls" on $\mathcal{B}_{p}$ where the local velocity approaches light speed $\Xi\left(\sigma^{\alpha}\right) \rightarrow 1$. If, as a result, $\mathcal{B}_{p}$ has the topology of a $p$ ball, with the $s^{n+1}$ shrinking to zero size at $\partial \mathcal{B}_{p}$, then the horizon topology is $S^{n+p+1}=S^{D-2}$.

The total area of the horizon is

$$
A_{\mathrm{H}}=\int_{\mathcal{B}_{p}} d V_{p} a_{\mathrm{H}}\left(\sigma^{\alpha}\right) .
$$

The first law of black-hole mechanics,

$$
\delta M=\frac{\kappa}{8 \pi G} \delta A_{\mathrm{H}}+\sum_{i} \Omega_{\mathrm{Hi}} \delta J_{i},
$$

can be seen to be satisfied by solutions of the blackfold equations [9].

Now we discuss some explicit solutions.

Products of odd-spheres. - Consider first a single odd sphere, $\mathcal{B}_{p}=S^{2 k+1}$, which we embed in a $2 k+$ 2-dimensional flat subspace of $\mathbb{R}^{D-1}$ with metric

$$
d \rho^{2}+\rho^{2} \sum_{i=1}^{k+1}\left(d \mu_{i}^{2}+\mu_{i}^{2} d \phi_{i}^{2}\right), \quad \sum_{i=1}^{k+1} \mu_{i}^{2}=1 .
$$

The sphere is embedded as $\rho=R$ and the world-volume spatial coordinates can be taken to be $k$ independent $\mu_{i}$ plus the $k+1$ Cartan angles $\phi_{i}$. Then we have $r_{i}=R \mu_{i}$. Assume now that all the angular velocities along the $\phi_{i}$ are equal in magnitude, $\left|\Omega_{\mathrm{H} i}\right|=\Omega_{\mathrm{H}}$. From (12) the boost velocity $\Xi=R \Omega_{\mathrm{H}}$ is uniform over the blackfold, and so is the thickness $r_{0}$. The blackfold equilibrium equations easily reduce to

$$
R=\sqrt{\frac{p}{n+p}} \frac{1}{\Omega_{\mathrm{H}}}
$$

(for $p=1$ we recover the result for black rings in [7]). The horizon geometry is $\mathcal{H}=S^{2 k+1} \times s^{n+1}$. One may also consider nonequal angular velocities. Then the radius $\rho=$ $R\left(\mu_{i}\right)$ depends nontrivially on $\mu_{i}$ and one must solve a second-order differential equation, which requires numerical analysis.

Consider now a product of odd spheres, $\mathcal{B}_{p}=$ $\prod_{p_{a} \in \text { odd }} S^{p_{a}}, p=\sum_{a} p_{a}$, embedded in a flat subspace of $\mathbb{R}^{D-1}$ with metric

$$
\sum_{a}\left(d \rho_{a}^{2}+\rho_{a}^{2} d \Omega_{p_{a}}^{2}\right) .
$$

Clearly, the total number of spheres cannot be larger than $D-1-p=n+2$. We look for blackfold geometries at constant radii $\rho_{a}=R_{a}$, with each odd-sphere rotating along all of its Cartan angles with angular velocities equal in magnitude to $\Omega_{\mathrm{H}}^{(a)}$. The equations of equilibrium factorize for each sphere and are solved for

$$
R_{a}=\sqrt{\frac{p_{a}}{n+p}} \frac{1}{\Omega_{\mathrm{H}}^{(a)}} .
$$

The horizon geometry is $\mathcal{H}=\prod_{p_{a} \in \text { odd }} S^{p_{a}} \times s^{n+1}$, and the mass, angular momenta, and area of the blackfold are easily obtained plugging these results in the general formulas above.

Ultraspinning Myers-Perry black holes as even-ball blackfolds.-The blackfold equations in a Minkowski background do not admit solutions for $\mathcal{B}_{p}$ a topological even sphere-the tension at fixed points of the rotation group cannot be counterbalanced by centrifugal forces. Instead they admit solutions where $\mathcal{B}_{p}$ is an ellipsoidal even ball, with thickness $r_{0}$ vanishing at the boundary of the ball so the horizon topology is $S^{D-2}$. These reproduce precisely all the physical properties of a Myers-Perry black hole with $p / 2$ ultraspins, which provides a highly nontrivial check on the approach. It also shows that the method remains sensible when the rotation has fixed points, in this case at the center of the ball. They also exemplify blackfolds with varying thickness $r_{0}\left(\sigma^{\alpha}\right)$.

We illustrate these solutions in the simplest nontrivial case of $p=2$, and postpone the general analysis to [9]. Consider a black twofold extending along a plane $d r^{2}+$ $r^{2} d \phi^{2}$ in Minkowski space. Being a plane, this $\mathcal{B}_{2}$ solves trivially the blackfold Eqs. (2). To set the blackfold in rotation along the $\phi$ axis we embed $\mathcal{B}_{2}$ as $\sigma^{1}=\phi, \sigma^{2}=$ $r$, with local boost along $\sigma^{1}$, and obtain $\Xi=r \Omega_{\mathrm{H}}$. Uniform $\Omega_{\mathrm{H}}$, i.e., rigid brane rotation, makes the local boost become lightlike at $r=\Omega_{\mathrm{H}}^{-1}$. Constancy of $\kappa$ implies that $r_{0}\left(r \rightarrow \Omega_{\mathrm{H}}^{-1}\right) \rightarrow 0$ so $\mathcal{B}_{2}$ becomes the disk $0 \leq$ $r \leq \Omega_{\mathrm{H}}^{-1}$. The physical magnitudes of the blackfold are

$$
\begin{gathered}
M=\left(\frac{n}{2 \kappa}\right)^{n} \frac{1}{\Omega_{\mathrm{H}}^{2}} \frac{n+3}{n+2}, \quad J=\left(\frac{n}{2 \kappa}\right)^{n} \frac{1}{\Omega_{\mathrm{H}}^{3}} \frac{2}{n+2}, \\
A_{\mathrm{H}}=2 \pi \Omega_{n+1}\left(\frac{n}{2 \kappa}\right)^{n+1} \frac{1}{\Omega_{\mathrm{H}}^{2}} \frac{1}{n+2} .
\end{gathered}
$$

If we now write $\Omega_{\mathrm{H}}=a^{-1}$ and $\frac{n}{2 \kappa}=r_{+}$, these equations 
reproduce exactly the values for an ultraspinning MyersPerry black hole in $D=n+5$ dimensions, with a single spin parameter $a$ and with horizon radial coordinate $r_{+}$, to leading order in $r_{+} / a$ [3]. The shape of the horizon is also accurately reproduced: for the ultraspinning black hole the thickness transverse to the rotation plane is $r_{+} \cos \theta$ [3], while for the blackfold, introducing $\theta=\arcsin \left(\Omega_{\mathrm{H}} r\right)$, we find thickness $r_{0}(\theta)=\frac{n}{2 \kappa} \cos \theta$, thus in perfect agreement. Also in both cases $a$ is the horizon radius in the plane parallel to the rotation. Observe that once the angular velocity and surface gravity are identified in the two constructions, there is no ambiguity when comparing physical magnitudes.

Minimal blackfolds.-For static blackfolds with space components of the stress tensor $\tau_{j}^{i}=-P \delta_{j}^{i}$, the blackfold equations reduce to $K^{\rho}=0$, where $K^{\rho}=h^{\mu \nu} K_{\mu \nu}^{\rho}$ is the mean curvature vector. Thus, $\mathcal{B}_{p}$ must span a (sufficiently regular and non-self-intersecting) minimal spatial submanifold. As far as we know, in Euclidean space no compact examples of these have been found.

Let us address some caveats about the blackfold approach. (i) One may worry whether the horizon of the black brane remains regular after bending its world volume. For black onefolds, i.e., thin black rings, Ref. [7] showed that this is the case iff the blackfold equations (2) are satisfied. An extension to black $p$ folds will be given elsewhere [9]. (ii) To leading order in $r_{0} / R$ the backreaction of the blackfold on the background geometry is neglected. It may happen that backreaction makes it impossible for a leading-order solution to remain stationaryby developing naked singularities revealing unbalanced forces, or more physically, by inducing evolution in time. This must be analyzed in a case-by-case basis, typically using physical input about the expected effects of selfgravitational attraction. Many minimal blackfolds likely exhibit this phenomenon. Such solutions would not correspond to actual stationary black holes, but they would still be of interest as blackfolds that evolve slowly (at least initially) with time scale small in $r_{0} / R$. (iii) Blackfolds may be (classically) unstable. Stability to long-wavelength $\left(\lambda \gg r_{0}\right)$ perturbations can be analyzed using the blackfold equations. There are, however, short-wavelength $(\lambda \sim$ $r_{0}$ ) instabilities, e.g., of Gregory-Laflamme type, outside the approach which would proceed on quick time scales, $\Gamma \sim 1 / r_{0}$.

We have presented the theory of neutral blackfolds, and examples have referred to stationary blackfolds in a Minkowski background. However, the method can be readily generalized [9] to charged blackfolds as well as other backgrounds (e.g., [10]) and some blackfold motions.

Our approach naturally organizes the dynamics of higher-dimensional neutral black holes according to the relative value of the lengths $(G M)^{1 / D-3}$ and $J / M$, where $J=\left(\sum_{i} J_{i}^{2}\right)^{1 / 2}$ : (i) $0 \leq J \lesssim M(G M)^{1 / D-3}$ : there is a single length scale on the horizon and the physics is qualitatively similar to the Kerr black hole. (ii) $J \sim M(G M)^{1 / D-3}$ : regime of mergers and connections between phases that occur when the two horizon scales meet, $r_{0} \sim R$. Such threshold phenomena occur outside the limit of validity of effective field theories and are very difficult to analyze, requiring extrapolations, new approaches or numerical analysis (also, some mergers may occur at large values of $J$ [7]). (iii) $J \gg M(G M)^{1 / D-3}$ : blackfolds. We have developed the tools to study the extremely rich physics in this regime. Rather than search for exact solutions for all possible higher-dimensional black holes, it seems to us more fruitful to study the dynamical features of blackfolds.

We thank E. Kiritsis and B. Kol for discussions. Hospitality by TIFR and ICTS (2008 Monsoon Workshop, Mumbai), KEK (Tsukuba), GGI (Firenze), and CERN TH Institute on Black holes, is acknowledged. R.E. was supported by DURSI 2005 SGR 00082, MEC FPA 2007-66665-C02, and CPAN CSD2007-00042 Consolider-Ingenio 2010. T. H. was supported by the Carlsberg foundation. V.N. was supported by an Individual Marie Curie Intra-European Grant and by ANR-05-BLAN-0079-02, MRTN-CT-2004-503369, and CNRS PICS No. 3059, No. 3747, and No. 4172. All authors were supported by MRTN-CT-2004-005104.

*emparan@ub.edu

†harmark@nbi.dk

*niarchos@cpht.polytechnique.fr

§obers@nbi.dk

[1] R. Emparan and H. S. Reall, Phys. Rev. Lett. 88, 101101 (2002); R. Emparan and H. S. Reall, Classical Quantum Gravity 23, R169 (2006).

[2] R. C. Myers and M. J. Perry, Ann. Phys. (N.Y.) 172, 304 (1986).

[3] R. Emparan and R. C. Myers, J. High Energy Phys. 09 (2003) 025.

[4] R. Gregory and R. Laflamme, Phys. Rev. Lett. 70, 2837 (1993); T. Harmark, V. Niarchos, and N.A. Obers, Classical Quantum Gravity 24, R1 (2007).

[5] T. Harmark, Phys. Rev. D 69, 104015 (2004); D. Gorbonos and B. Kol, J. High Energy Phys. 06 (2004) 053.

[6] W. D. Goldberger and I.Z. Rothstein, Phys. Rev. D 73, 104029 (2006); Y.Z. Chu, W.D. Goldberger, and I.Z. Rothstein, J. High Energy Phys. 03 (2006) 013; B. Kol and M. Smolkin, Phys. Rev. D 77, 064033 (2008).

[7] R. Emparan, T. Harmark, V. Niarchos, N. A. Obers, and M. J. Rodriguez, J. High Energy Phys. 10 (2007) 110.

[8] B. Carter, Int. J. Theor. Phys. 40, 2099 (2001).

[9] R. Emparan, T. Harmark, V. Niarchos, and N. A. Obers (to be published).

[10] M. M. Caldarelli, R. Emparan, and M. J. Rodriguez, J. High Energy Phys. 11 (2008) 011; J. Camps, R. Emparan, P. Figueras, S. Giusto, and A. Saxena, J. High Energy Phys. 02 (2009) 021. 